\title{
Value of BRAF V600E in High-Risk Thyroid Nodules with Benign Cytology Results
}

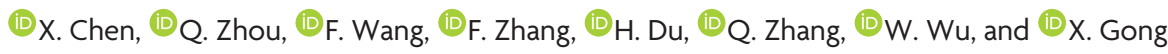

\begin{abstract}
BACKGROUND AND PURPOSE: Limitations of ultrasound-guided fine-needle aspiration include nondiagnostic, indeterminate cytology and false-negative results. The BRAFV600E mutation is a specific biomarker for papillary thyroid carcinoma. This study aimed to investigate the additional diagnostic role of the BRAF V600E mutation in high-risk thyroid nodules with benign cytology results.
\end{abstract}

MATERIALS AND METHODS: A total of 787 high-risk nodules in 720 patients underwent ultrasound-fine-needle aspiration. A subsequent BRAF V600E mutation test was performed on thyroid nodules with benign cytology. Final pathology confirmed thyroid nodules with benign cytology that were positive for the BRAF V600E mutation. Ultrasound was performed on thyroid nodules with benign cytology results that were negative for the BRAF V600E mutation. Fine-needle aspiration was repeated on thyroid nodules with enlarged size or changed ultrasound features.

RESULTS: Among the 787 nodules, 292 thyroid nodules had benign cytology results with 256 nodules negative for the BRAF V600E mutation and 36 nodules positive for the BRAFV $600 E$ mutation. Thirty-one nodules positive for the BRAF V600E mutation were confirmed malignant, and 5 nodules were confirmed benign by pathology. Fine-needle aspiration was repeated on 11 enlarged thyroid nodules with benign cytology findings that were negative for the BRAF V600E mutation. The results of repeat fine-needle aspiration were 4 benign nodules, 2 follicular neoplasms or suspected follicular neoplasms, 3 suspected malignancies, and 2 malignant nodules. Among the 36 thyroid nodules positive for the BRAF V600E mutation, 25 (69.4\%) had $\geq 2$ suspicious ultrasound features and 11 (30.6\%) nodules had 1 suspicious ultrasound feature.

CONCLUSIONS: The BRAF V600E mutation test can detect papillary thyroid carcinomas that might be missed by fine-needle aspiration. We recommend that fine-needle aspiration be routinely accompanied by the BRAF V600E mutation test in high-risk thyroid nodules with $\geq 2$ suspicious ultrasound features.

ABBREVIATIONS: FNA = fine-needle aspiration; $\mathrm{PCR}=$ polymerase chain reaction; PTC = papillary thyroid carcinoma; US = ultrasound

T hyroid nodules are commonly found in clinical practice. Although most thyroid nodules are benign, the differentiation between malignant and benign lesions remains a challenge for clinicians. Ultrasound (US) has been established as the first detection tool in thyroid studies. ${ }^{1}$ The expected malignancy risk of high-risk thyroid nodules is $50 \%-90 \%$ based on the presence of $\geq 1$ suspicious finding. ${ }^{2}$ Fine-needle aspiration (FNA) cytology

Received May 8, 2018; accepted after revision September 29.

From the Departments of Endocrinology and Metabolism (X.C., Q. Zhou, W.W., X.G.), Pathology (F.W., Q. Zhang), and Ultrasound (H.D.), The First Affiliated Hospital of Wenzhou Medical University, Zhejiang Province, China; and Department of Medicine (F.Z.), School of Renji College, Wenzhou Medical University, Wenzhou, China.

Xiaojun Chen, Qi Zhou, Fang Wang, and Fangfei Zhang contributed equally to this work.

This work was supported by the Medical Science and Technology Project of Zhejiang Province (grant no. 2018KY510) and the Science and Technology Project of Wenzhou (grant No. Y20170046). represents the criterion standard for determining the nature of thyroid nodules. ${ }^{2,3}$ Fine-needle aspiration is a reliable method with good sensitivity and specificity. However, the limitations of ultrasound-guided fine-needle aspiration include nondiagnostic, indeterminate cytology and false-negative and falsepositive results, which are $10 \%-40 \%$ of all cytology results. ${ }^{4-6}$ The conventional practice is US monitoring or repeat FNA when a mismatch is determined between radiology and cytology findings. ${ }^{2,3}$

Molecular testing of thyroid fine-needle aspirates has emerged as a tool to complement routine cytopathologic examinations. ${ }^{7,8}$ The BRAF V600E mutation is used as a specific biomarker for

Please address correspondence to Xiaohua Gong, MD, Department of Endocrinology, The First Affiliated Hospital of Wenzhou Medical University, Ouhai District, Wenzhou 325015, Zhejiang Province, China; e-mail: gxh1204@163.com

-- Indicates open access to non-subscribers at www.ajnr.org

http://dx.doi.org/10.3174/ajnr.A5898 
papillary thyroid carcinoma (PTC) and has a high positive predictive value $(95.5 \%-100.0 \%) .^{9,10}$

Considering the false-negative rate of $3.6 \%-21 \%$ in USFNA $^{11,12}$ and the high-risk rate of malignancy in thyroid nodules with suspicious US features, this study investigated the additional diagnostic role of the BRAF V600E mutation in high-risk thyroid nodules with benign cytology findings and compared the clinical US features of thyroid nodules with benign cytology results and tests positive for the BRAF V600E mutation with nodules that had tests negative for the BRAF V600E mutation.

\section{MATERIALS AND METHODS}

This was a prospective study. It was approved by the Ethics Committee of The First Affiliated Hospital of Wenzhou Medical University. All study participants provided written informed consent before undergoing US-FNA and BRAF V600E mutation tests. The study conformed to the Declaration of Helsinki.

\section{Patients}

A total of 787 high-risk thyroid nodules in 720 patients underwent US-FNA from October 2015 to March 2017 in our hospital. On the basis of the Bethesda System for Reporting Thyroid Cytopathology, FNA cytology results were categorized as nondiagnostic, benign, atypia of undetermined significance/follicular lesion of undetermined significance, follicular neoplasm or suspicious follicular neoplasm, suspected malignancy, and malignant. A subsequent BRAF V600E mutation test was performed on 292 patients with benign cytology results of thyroid nodules. A total of 36 thyroid nodules were positive for the BRAF V600E mutation, and 256 thyroid nodules were negative for the BRAF V600E mutation. The 36 thyroid nodules positive for the BRAF V600E mutation underwent an operation. US monitoring was performed on thyroid nodules with benign cytology results and negative for the $B R A F$ V600E mutation every 6 months. The follow-up duration for patients was $>1$ year. Repeat fine-needle aspiration biopsy was conducted on thyroid nodules that met at least 1 of the following criteria: The nodule grew during follow-up $(>50 \%$ change in volume or a $20 \%$ increase in at least 2 nodule dimensions with a minimal increase of $2 \mathrm{~mm}$ in solid nodules or in the solid portion of mixed cystic-solid nodules). The patients underwent an operation if the results of repeat cytology were follicular neoplasm or suspicious follicular neoplasm, suspected malignancy, or malignant, and continued follow-up US every 6 months if the results of repeat cytology were nondiagnostic, benign, or atypia of undetermined significance/follicular lesion of undetermined significance.

\section{US and US-FNA}

Two radiologists with 10 years of experience in thyroid imaging performed US examinations using a 5- to $12-\mathrm{MHz}$ linear array transducer (EZU-MT28-S1; Hitachi Medical, Tokyo, Japan). Radiologists interpreted US findings on the basis of the internal composition, echogenicity, margin, calcification, and shape. Subsequently recorded US features were reclassified on the basis of the updated American Association of Clinical Endocrinologists, American College of Endocrinology, and Associazione Medici Endocrinologi guidelines. ${ }^{2}$ Highly suspicious US features indicative of thyroid cancer included $\geq 1$ of the following features: marked hypoechogenicity (confer prethyroid muscles), spiculated or microlobulated margins, microcalcifications, taller-than-wide shape, evidence of extrathyroidal growth, or pathologic adenopathy. ${ }^{2}$

FNAs were performed under US guidance with a 23 -ga needle attached to a 2-mL syringe. Each lesion was aspirated in 2-3 passes in different directions. The aspirates were expelled on glass slides, smeared, and immediately placed in 95\% alcohol and were sent for cytologic analysis by 2 experienced cytologists. The 1 remaining pass of material was rinsed in $180-\mu \mathrm{L}$ cytolysis liquid and was stored at $-20^{\circ} \mathrm{C}$ for subsequent $B R A F$ V600E mutation testing.

\section{DNA Isolation and BRAF V600E Detection}

$B R A F$ V600E mutation analysis was performed on the remaining extracted DNA from FNA cells after cytologic evaluation. DNA extraction was successfully completed in all samples following the manufacturer's instructions with a commercially available kit (ADx-Amplification Refractory Mutation System [ADx-ARMS]; AmoyDX, Xiamen, China). The quantity of isolated DNA was assessed using a NanoDrop2000 spectrophotometer (Thermo Fisher Scientific, Waltham, Massachusetts, USA). All samples in this study were adequate for genetic detection. The samples were analyzed by applying the ADx-ARMS technique. Briefly, each polymerase chain reaction (PCR) mixture contained $5 \mu \mathrm{L}$ of extracted DNA and other chemicals available in a kit (ADx-ARMS; AmoyDX) that contained oligonucleotide primers, Taq DNA polymerase, oligonucleotide probes, nucleotides, and buffers. PCR reaction was conducted on a qRT-PCR machine (ABI 7500; Applied Biosystems, Foster City, California) with an initial denaturation step at $94^{\circ} \mathrm{C}$ for 5 minutes, 15 annealing cycles at $95^{\circ} \mathrm{C}$ for 25 seconds, $64^{\circ} \mathrm{C}$ for 20 seconds, and $72^{\circ} \mathrm{C}$ for 20 seconds, followed by 31 extension cycles at $93^{\circ} \mathrm{C}$ for 25 seconds, $60^{\circ} \mathrm{C}$ for 35 seconds, and $72^{\circ} \mathrm{C}$ for 20 seconds.

\section{Statistical Analysis}

The comparison of variables between the 2 groups was analyzed with Student $t$ and Mann-Whitney $U$ tests. Qualitative variables were evaluated with a $\chi^{2}$ test. Analyses were performed with Statistical Package for Social Sciences, Version 19.0 (IBM, Armonk, New York). A confidence interval of $95 \%$ and a significance level of $5 \%$ were used.

\section{RESULTS}

A total of 787 high-risk nodules in 720 patients (653 patients had a single nodule and 67 patients had 2 nodules; 511 women, 209 men; mean age, $46.2 \pm 11.7$ years; range, $15-83$ years) were included in the study and underwent US-FNA. A subsequent $B R A F$ V600E mutation test was performed on 292 patients with benign cytology results. A total of 36 thyroid nodules with benign cytology results were positive for the BRAF V600E mutation and 256 thyroid nodules with benign cytology results were negative for the $B R A F$ V600E mutation. Figure 1 represents the clinical course of 292 thyroid nodules with benign cytology results. Of the 36 thyroid nodules with benign cytology results and positive for the $B R A F$ V600E mutation, 10 were in men and 26 were in women. The mean age of patients was $44.7 \pm 11.3$ years and ranged from 18 to 75 years. The mean size of the thyroid nodules was $9.4 \mathrm{~mm}$

AJNR Am J Neuroradiol 39:2360-65 Dec 2018 www.ajnr.org 
and ranged from 5.3 to $25 \mathrm{~mm}$. All 36 patients underwent thyroidectomy. Nodules in 31 of 36 patients were confirmed malignant by surgical pathology. Twelve nodules were PTC, and 19 were papillary thyroid microcarcinomas (Fig 2). Twenty-three of 36 nodules measured $<1 \mathrm{~cm}$, whereas the remaining 13 were $>1$ $\mathrm{cm}$ (Table 1). Among the patients, 11 had 1 suspicious US feature, 17 had 2 suspicious US features, 7 had 3 suspicious US features, and 1 had 4 suspicious US features (Table 1). Among the 256 patients with thyroid nodules with benign cytology results and negative for the BRAF V600E mutation, 27 were men and 229 were women. The mean age of patients was $47.3 \pm 13.9$ years and ranged from 15 to 83 years. The mean size of thyroid nodules was $11.8 \mathrm{~mm}$ and ranged from 5.7 to $39 \mathrm{~mm}$ (Table 1). Eleven patients had enlarged nodules during follow-up and underwent repeat FNA. Nodules of 4 patients were still benign during repeat FNA, and the patients continued follow-up US. Two patients had fol-

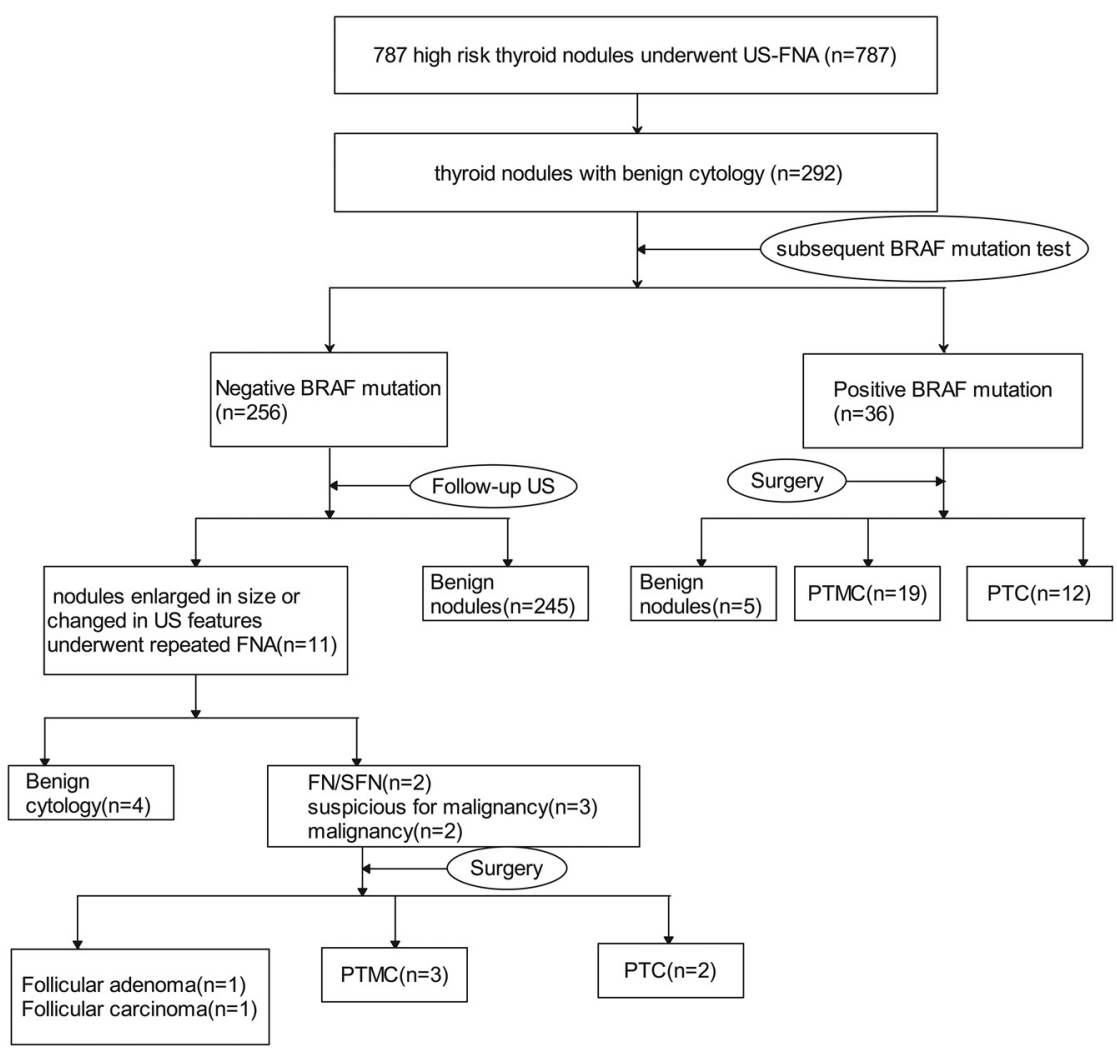

FIG 1. Diagram of the study group. PTMC indicates papillary thyroid microcarcinoma. licular neoplasms or suspicious follicular neoplasms, 3 nodules were suspicious for malignancy, and 2 patients with malignancy on repeat FNA underwent an operation. One of the 7 nodules was confirmed as a follicular adenoma, one was follicular thyroid carcinoma, and the other 5 were confirmed as PTC based on pathology.

No statistically significant differences were observed in mean age, mean size, and US features such as echogenicity and margins between the nodules positive for the BRAF V600E mutation and nodules negative for the BRAF V600E mutation (Table 1). Statistically significant differences were observed in sex, the number of suspicious US features, and other US features such as calcification and shape between the 2 groups (Table 1 ).

Five of the 36 thyroid nodules with benign cytology results and positive for the BRAF V600E mutation had benign results on surgical pathology (Table 2). Among the patients, 2 nodules were confirmed as nodular goiter with underlying lymphocytic thyroiditis (Fig 3), 2 nodules were nodular goiter, and 1 was a fibrotic nodule with calcification. Three of 5 nodules measured $<1 \mathrm{~cm}$, whereas the remaining 2 were $>1 \mathrm{~cm}$. Among the patients, 2 had 1 suspicious US feature, 2 had 2 suspicious US features, and 1 had 3 suspicious US features.

\section{DISCUSSION}

Sonography is a sensitive available technique that enables clinicians to detect thyroid nodules with their dimensions and sonographic features suggestive of malignancy. According to the 2016 American Association of Clinical Endocrinologists, American College of Endocrinology, and Associazione Medici Endocrinologi guidelines, the estimated risks of malignancy were $50 \%-90 \%$ for thyroid nodules with highly suspicious US features. ${ }^{2}$

FNA is currently the reliable nonsurgical approach for the diagnosis of thyroid nodules. However, thyroid FNA is not an accurate test. A review of the lit-
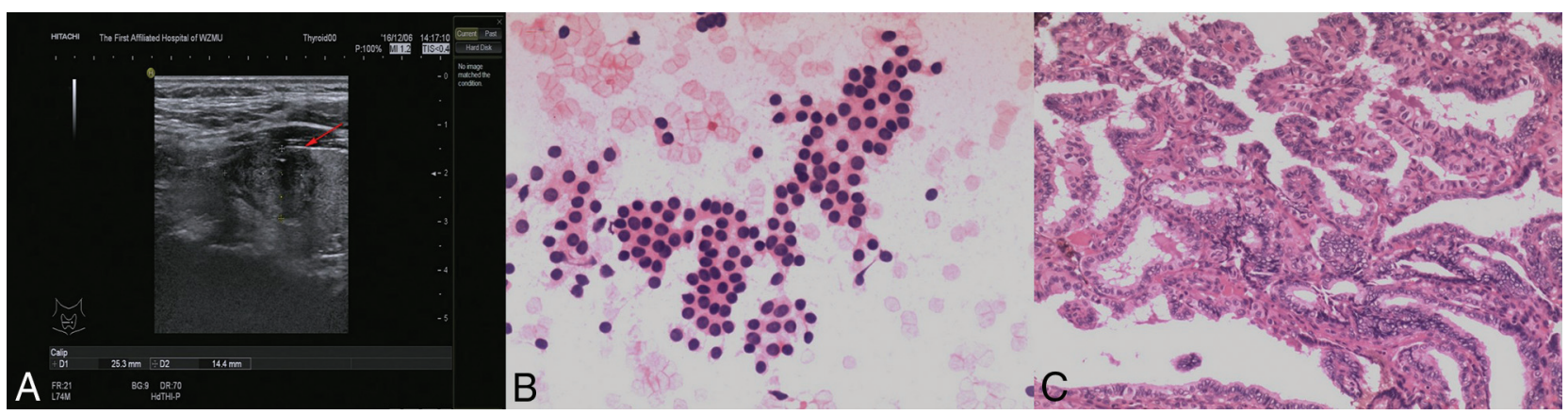

FIG 2. Right thyroid with a palpable lesion in a 20-year-old man. US scan of the right thyroid reveals a 25-mm hypoechoic nodule (arrow) with an irregular margin and microcalcification (A). The cytologic diagnosis from US-FNA is benign (B, HE stain, magnification 4X10) and positive for the BRAF V600E mutation. The nodule is confirmed as PTC by surgical pathology (C, HE stain, magnification 10X10). 
erature revealed thyroid nodules with overall false-negative rates in FNA (malignant histology of a nodule with benign cytology) that ranged from $<1 \%$ to $12 \% .^{13,14}$ Other studies have observed higher false-negative rates in nodules of $\geq 3$ or $4 \mathrm{~cm}(10 \%-13 \%$ in larger nodules compared with $5 \%-6 \%$ in smaller nodules). ${ }^{15,16}$ By contrast, Shrestha et $\mathrm{al}^{13}$ reported that false-negative rates for FNA were $7.0 \%$ overall, $15.8 \%$ in nodules that ranged between 0.5 and $0.9 \mathrm{~cm}, 6.3 \%$ in nodules that ranged between 1.0 and $3.9 \mathrm{~cm}$, and $7.1 \%$ in nodules of $\geqq 4.0 \mathrm{~cm}$. Shrestha's study showed a tendency toward a high false-negative rate in subcentimeter nodules. False-negative results complicated the subsequent clinical management of patients. The conventional practice was US monitoring or repeat FNA when a mismatch occurred between radiology and cytology findings.

The BRAF V600E mutation is commonly found in PTC. In China, its mutation rate is up to $69 \%-85.3 \%$ in patients with PTC. ${ }^{10,17}$ The BRAF V600E mutation analysis has proved valuable and accurate as an adjunctive diagnostic tool on US-FNA in providing additional information in the differential diagnosis of

\begin{tabular}{|c|c|c|c|}
\hline & $\begin{array}{l}\text { Positive for } \\
\text { BRAF V600E } \\
\text { Mutation }\end{array}$ & $\begin{array}{c}\text { Negative for } \\
\text { BRAF V600E } \\
\text { Mutation }\end{array}$ & $P$ \\
\hline No. of nodules & 36 & 256 & \\
\hline Diagnosis & & & $<.001$ \\
\hline Malignant & 31 & 6 & \\
\hline Benign & 5 & 250 & \\
\hline Age (mean) (yr) & $44.7 \pm 11.3$ & $47.3 \pm 13.9$ & .31 \\
\hline Sex & & & $<.001$ \\
\hline $\mathrm{F}$ & 2 & 229 & \\
\hline$M$ & 10 & 27 & \\
\hline Mean size of nodules (mm) & $9.4 \pm 3.7$ & $11.8 \pm 4.1$ & .41 \\
\hline $5-10$ & 23 & 145 & \\
\hline$>10$ & 13 & 112 & \\
\hline No. of suspicious US features & & & $<.001$ \\
\hline 1 & 11 & 243 & \\
\hline 2 & 17 & 12 & \\
\hline 3 & 7 & 1 & \\
\hline 4 & 1 & 0 & \\
\hline Echogenicity & & & .11 \\
\hline Hyper- or isoechoic & 1 & 15 & \\
\hline Hypoechoic & 30 & 229 & \\
\hline Marked hypoechoic & 5 & 12 & \\
\hline Margin & & & .18 \\
\hline Circumscribed & 15 & 137 & \\
\hline Not circumscribed & 21 & 119 & \\
\hline Calcification & & & $<.001$ \\
\hline No calcifications & 12 & 161 & \\
\hline Microcalcification & 21 & 72 & \\
\hline Macrocalcification & 3 & 23 & \\
\hline Shape & & & $<.01$ \\
\hline Parallel & 16 & 167 & \\
\hline Taller-than-wide & 20 & 89 & \\
\hline Evidence of extrathyroidal growth & 0 & 0 & \\
\hline Pathologic adenopathy & 2 & 0 & \\
\hline
\end{tabular}

PTC that shows nondiagnostic or indeterminate cytology results. ${ }^{8,18-20}$ However, what role does the BRAF V600E mutation analysis play in thyroid nodules with highly suspicious US features and negative FNA findings? A review of the literature revealed that few studies focused on the role of molecular testing in false-negative FNA results. Proietti et $\mathrm{al}^{21}$ reported 54 cases of false-negative cytology results among 1347 PTCs. BRAF V600E mutations were found in 6 cases $(11 \%)$, and Ras alterations were present in 16 cases $(29.6 \%)$. Consequently, the authors suggested that preoperative molecular assessment was valuable for benign nodules. Kim et $\mathrm{al}^{22}$ observed 31 nodules with benign cytology findings and positive for the BRAF V600E mutation. Among the cases, 17 underwent thyroidectomy. Fifteen of 17 nodules were malignant, and 2 were benign. However, 14 nodules were not confirmed with resection, and whether the cytology results were false-negative or the BRAF V600E mutation results were false-positive was undetermined.

In our prospective study, we observed 292 patients who had high-risk thyroid nodules with benign cytology findings who underwent subsequent BRAF V600E mutation testing. Among them, 256 patients had nodules negative for the BRAF V600E mutation and 36 patients had nodules positive for the BRAF V600E mutation. Thirty-six patients who had nodules with highly suspicious US features and were positive for the BRAF V600E mutation underwent thyroidectomy. Nodules of thirty-one of 36 patients were confirmed malignant by surgical pathology, and $B R A F$ V600E mutation analysis decreased the false-negative rate of FNA. These results supported the high specificity of the $B R A F$ V600E mutation $(31 / 36,86.1 \%)$ in thyroid nodules, and $B R A F$ V600E analysis played a role in thyroid nodules that showed highly suspicious US features with benign cytology results. Among the 31 cases confirmed by pathology, 22 (71.0\%) cases had $\geq 2$ suspicious US features and 9 (29.0\%) had 1 suspicious US feature. Follow-up US was performed in 256 patients who had thyroid nodules with benign cytology results and were negative for the BRAF V600E mutation. During the follow-up, 11 patients had enlarged nodules and underwent repeat FNA. Nodules of 4 patients were still benign on repeat FNA, and the 7 remaining patients underwent an operation, in which 2 nodules were follicular neoplasm or suspicious follicular neoplasm, 3 were suspicious for malignancy, and 2 were malignant on repeat FNA. One of the 7 nodules was confirmed as follicular adenoma, 1 was follicular thyroid carcinoma, and the other 5 were confirmed as PTC on the basis of pathology. On the basis of our results, we recommended diagnostic lobectomy in the management of thyroid nodules that showed highly suspicious US features, especially for a nodule with $\geq 2$ suspicious US features with benign cytology results but positive for the BRAF V600E mutation. However, we determined that results negative for the BRAF V600E mutation

Table 2: Demographics of 5 patients whose nodules had benign cytology results and were positive for the BRAF V600E mutation

\begin{tabular}{llcccl}
\hline No. & $\begin{array}{l}\text { Age } \\
\text { (yr) }\end{array}$ & Sex & $\begin{array}{c}\text { No. of Suspicious } \\
\text { US Features }\end{array}$ & $\begin{array}{c}\text { Size of the } \\
\text { Nodules (mm) }\end{array}$ & Pathology Results \\
\hline 1 & 48 & F & 1 & 5.3 & Nodule goiter \\
2 & 39 & F & 2 & 11 & Fibrotic nodule with calcification \\
3 & 45 & M & 2 & 9 & Nodule goiter \\
4 & 45 & M & 1 & 6 & Nodule goiter with underlying lymphocytic thyroiditis \\
5 & 69 & F & 3 & 13 & Nodule goiter with underlying lymphocytic thyroiditis \\
\hline
\end{tabular}



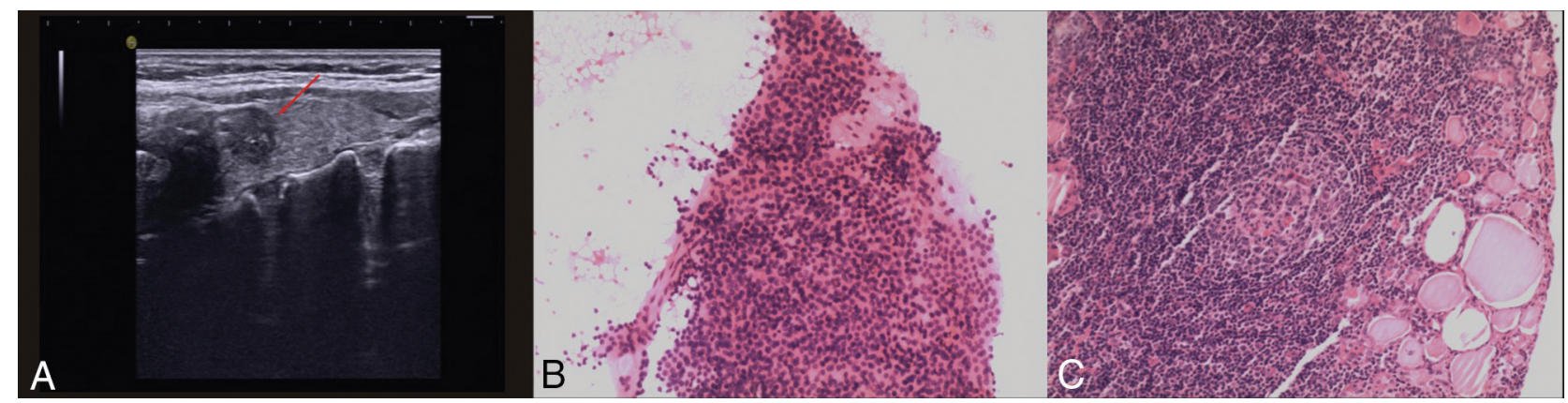

FIG 3. An incidentally found nodule in a 69-year-old woman during US screening. US scans reveal a 13-mm hypoechoic nodule (arrrow) with microcalcification in the right thyroid $(A)$. The cytologic diagnosis from US-FNA is benign ( $B$, HE stain, magnification 4 X10) and positive for the $B R A F$ V600E mutation using direct DNA sequencing. The nodule is confirmed as a nodular goiter with underlying lymphocytic thyroiditis ( $C$, HE stain, magnification 10X10).

did not rule out malignancy with benign cytology results. Five cases of PTC and 1 follicular thyroid carcinoma were missed by FNA and BRAF V600E mutation tests.

In our institution, BRAF V600E mutation analysis was performed using ADx-ARMS, which is a real-time PCR. Real-time PCR was more sensitive than traditional methods such as DNA sequencing and pyrosequencing because real-time PCR detected the BRAF V600E mutation in a small amount of mutant DNA. ${ }^{23-25}$ High-sensitivity ADx-ARMS method suggested its diagnostic significance for detecting the BRAF V600E mutation in thyroid samples. Studies of highly sensitive diagnostic methods for detecting the BRAF V600E mutation have rarely reported false-positive mutations in the literature. Kim et $\mathrm{al}^{19}$ reported 5 false-positive cases in 2010, and DiLorenzo et $\mathrm{al}^{26}$ reported 1 falsepositive case. The 2 studies stated that the sensitive BRAF V600E molecular testing using dual priming oligonucleotide-based multiplex PCR was the cause of the false-positive results. Kim et $\mathrm{al}^{23}$ also compared real-time PCR and pyrosequencing for the detection of the BRAF V600E mutation in FNA; 6 false-positive cases were detected by real-time PCR, whereas 4 false-positive cases were detected by pyrosequencing. We also observed 5 falsepositive cases among the 36 nodules treated by thyroidectomy. Two were confirmed as nodular goiter with underlying lymphocytic thyroiditis, 2 were nodular goiter, and 1 was a fibrotic nodule with calcification by surgical pathology.

\section{CONCLUSIONS}

The BRAF V600E mutation test detected PTC missed by FNA. We recommend that fine-needle aspiration be routinely accompanied by BRAF V600E mutation testing in high-risk thyroid nodules with $\geq 2$ suspicious US features. The combination of $B R A F$ V600E mutation testing and cytologic diagnosis improved the sensitivity and accuracy of detection.

\section{ACKNOWLEDGMENTS}

We acknowledge our colleagues from the Endocrinology and Pathology Departments at The First Affiliated Hospital of Wenzhou Medical University for their assistance in the present study.

\section{REFERENCES}

1. Sipos JA. Advances in ultrasound for the diagnosis and management of thyroid cancer. Thyroid 2009;19:1363-72 CrossRef Medline
2. Gharib H, Papini E, Garber JR, et al; AACE/ACE/AME Task Force on Thyroid Nodules. American Association of Clinical Endocrinologists, American College of Endocrinology, and Associazione Medici Endocrinologi Medical Guidelines for Clinical Practice for the Diagnosis and Management of Thyroid Nodules: 2016 Update. Endocr Pract 2016;22:622-39 CrossRef Medline

3. Haugen BR, Alexander EK, Bible KC, et al. 2015 American Thyroid Association Management. Thyroid 2016;26:1-133 CrossRef Medline

4. Yoon JH, Moon HJ, Kim E-K, et al. Inadequate cytology in thyroid nodules: should we repeat aspiration or follow-up? Ann Surg Oncol 2011;18:1282-89 CrossRef Medline

5. Yoon JH, Kim EK, Moon HJ, et al. Is follow-up BRAF(V600E) mutation analysis helpful in the differential diagnosis of thyroid nodules with negative results on initial analysis? PLoS One 2013;8: e58592 CrossRef Medline

6. Moon HJ, Son E, Kim EK, et al. The diagnostic values of ultrasound and ultrasound-guided fine needle aspiration in subcentimetersized thyroid nodules. Ann Surg Oncol 2012;19:52-59 CrossRef Medline

7. Kim SK, Hwang TS, Yoo YB, et al. Surgical results of thyroid nodules according to a management guideline based on the $\mathrm{BRAF}(\mathrm{V} 600 \mathrm{E})$ mutation status. J Clin Endocrinol Metab 2011;96:658-64 CrossRef Medline

8. Moon HJ, Kim E-K, Chung WY, et al. Diagnostic value of BRAF(V600E) mutation analysis of thyroid nodules according to ultrasonographic features and the time of aspiration. Ann Surg Oncol 2011;18:792-99 CrossRef Medline

9. Koh J, Choi JR, Han KH, et al. Proper indication of BRAF(V600E) mutation testing in fine-needle aspirates of thyroid nodules. PLoS One 2013;8:e64505 CrossRef Medline

10. Zhang Y, Xu T, Cui D, et al. Value of TIRADS, BSRTC and FNABRAF V600E mutation analysis in differentiating high-risk thyroid nodules. Sci Rep 2015;5:16927 CrossRef Medline

11. Lew JI, Snyder RA, Sanchez YM, et al. Fine needle aspiration of the thyroid: correlation with final histopathology in a surgical series of 797 patients. J Am Coll Surg 2011;213:188-94; discussion 194-95 CrossRef Medline

12. Yeh MW, Demircan O, Ituarte P, et al. False-negative fine-needle aspiration cytology results delay treatment and adversely affect outcome in patients with thyroid carcinoma. Thyroid 2004;14: 207-15 CrossRef Medline

13. Shrestha M, Crothers BA, Burch HB. The impact of thyroid nodule size on the risk of malignancy and accuracy of fine-needle aspiration: a 10-year study from a single institution. Thyroid 2012; 22:1251-56 CrossRef Medline

14. Cavallo A, Johnson DN, White MG, et al. Thyroid nodule size at ultrasound as a predictor of malignancy and final pathologic size. Thyroid 2017;27:641-50 CrossRef Medline

15. McCoy KL, Jabbour N, Ogilvie JB, et al. The incidence of cancer and rate of false-negative cytology in thyroid nodules greater than or 
equal to $4 \mathrm{~cm}$ in size. Surgery 2007;142:837-44; discussion 844 .e1-3 CrossRef Medline

16. Agcaoglu O, Aksakal N, Ozcinar B, et al. Factors that affect the falsenegative outcomes of fine-needle aspiration biopsy in thyroid nodules. Int J Endocrinol 2013;2013:126084CrossRef Medline

17. Lu ZZ, Zhang Y, Wei SF, et al. Outcome of papillary thyroid microcarcinoma: study of 1,990 cases. Mol Clin Oncol 2015;3:672-76 CrossRef Medline

18. Cohen Y, Rosenbaum E, Clark DP, et al. Mutational analysis of BRAF in fine needle aspiration biopsies of the thyroid: a potential application for the preoperative assessment of thyroid nodules. Clin Cancer Res 2004;10:2761-65 CrossRef Medline

19. Kim SW, Lee JI, Kim JW, et al. BRAFV600E mutation analysis in fine-needle aspiration cytology specimens for evaluation of thyroid nodule: a large series in a BRAFV600E-prevalent population. J Clin Endocrinol Metab 2010;95:3693-700 CrossRef Medline

20. Xing M, Tufano RP, Tufaro AP, et al. Detection of BRAF mutation on fine needle aspiration biopsy specimens: a new diagnostic tool for papillary thyroid cancer. J Clin Endocrinol Metab 2004;89: 2867-72 CrossRef Medline

21. Proietti A, Borrelli N, Giannini R, et al. Molecular characteriza- tion of $\mathbf{5 4}$ cases of false-negative fine-needle aspiration among 1347 papillary thyroid carcinomas. Cancer Cytopathol 2014;122: 751-59 CrossRef Medline

22. Kim SY, Kim EK, Kwak JY, et al. What to do with thyroid nodules showing benign cytology and $\mathrm{BRAF}(\mathrm{V} 600 \mathrm{E})$ mutation? A study based on clinical and radiologic features using a highly sensitive analytic method. Surgery 2015;157:354-61 CrossRef Medline

23. Kim WY, Kim H, Hwang TS. Comparison between real-time PCR and pyrosequencing for detection of BRAF V600E mutation in thyroid fine-needle aspirates. Appl Immunohistochem Mol Morphol 2017;25:358 - 65 CrossRef Medline

24. Ellison G, Donald E, McWalter G, et al. A comparison of ARMS and DNA sequencing for mutation analysis in clinical biopsy samples. $J$ Exp Clin Cancer Res 2010;29:132 CrossRef Medline

25. Huang T, Zhuge J, Zhang WW. Sensitive detection of BRAF V600E mutation by Amplification Refractory Mutation System (ARMS)PCR. Biomarker Res 2013;1:3 CrossRef Medline

26. DiLorenzo M, Miller J, Tuluc M, et al. False-positive FNA due to highly sensitive BRAF assay. Endocr Pract 2014;20:e8-10 CrossRef Medline 